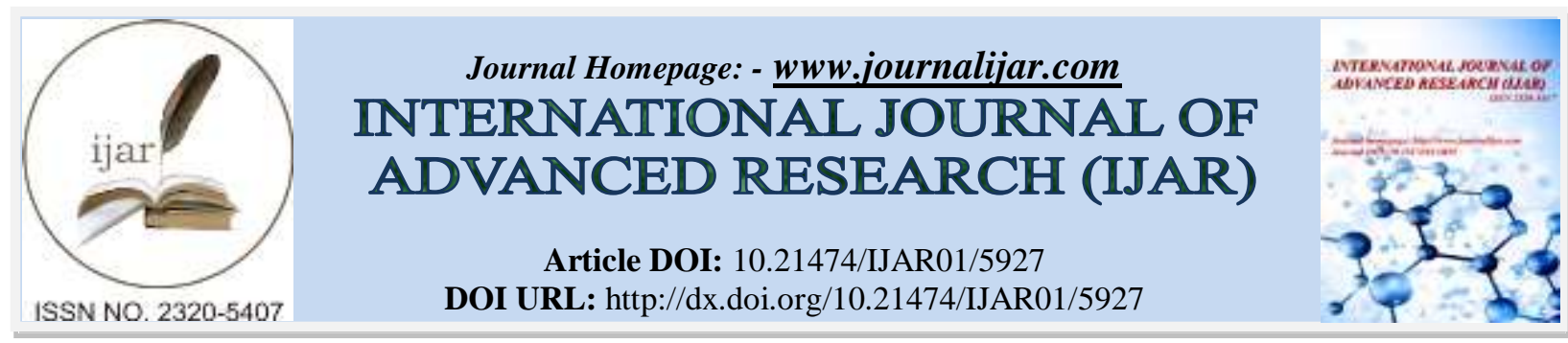

RESEARCH ARTICLE

\title{
COMPARISON OF ACCURACY IN AGE ESTIMATION BY MODIFIED KVAAL'S FORMULA AND CORONAL PULP CAVITY INDEX- A RADIOGRAPHIC STUDY.
}

Dr. Pushpa ${ }^{1}$, Dr. Vaishali Keluskar ${ }^{2}$, Dr. Anjana Bagewadi ${ }^{3}$ and Dr. Alka Kale ${ }^{4}$.

1. Post graduate, MDS in Oral Medicine and Radiology, KLE V.K. Institute of Dental Sciences, Belagavi, Karnataka, India.

2. Professor, Department of Oral Medicine and Radiology, KLE V.K. Institute of Dental Sciences, Belagavi, Karnataka, India.

3. Head and Professor, Department of Oral Medicine and Radiology, KLE V.K. Institute of Dental Sciences, Belagavi, Karnataka, India.

4. Principal, Department of Oral Pathology, KLE V.K. Institute of Dental Sciences, Belagavi, Karnataka, India.

\section{Manuscript Info}

Manuscript History

Received: 21 September 2017

Final Accepted: 23 October 2017

Published: November 2017

Key words:-

Age estimation, modified Kvaal's formula, coronal pulp cavity index(CPCI), maxillary second premolar, periapical digital radiographs, paralleling technique.

\section{Abstract}

Background: Age determination has become increasingly important in forensic science not only for the identification of corpses, but also to clarify legal queries in delineating juvenile and young adults in spheres pertaining to employment, labour acts and criminal offences. It plays a crucial role in forensic medicine, especially in connection with crimes and accidents.

Aim: To estimate the accuracy of age by using modified Kvaals formula and Coronal pulp cavity index.

Objectives:

1. To assess the accuracy of modified Kvaal's formula and Coronal pulp cavity index in estimating age among Indian population.

2. To compare the standard error of estimated age among both technique.

3. To establish the standard technique in assessing age among 5 groups.

Methodology: 100 individuals were selected within age group of 20-70 years. Digital intraoral periapical radiographs was taken by using KODAK 8000 RVG machine with the exposure factors of $65 \mathrm{KVp}$ and $8 \mathrm{~mA}$ for $0.2 \mathrm{~s}$ for either right or left side of maxillary second premolar by paralleling technique and maximum length of tooth, root and pulp were measured based on Modified KVAAL's method and CPCI using the KODAK Dental Imaging software.

Statistical analysis: The results obtained will be analyzed by using Descriptive analysis, Chi square test and ANOVA

Results: Modified kvaals formula and CPCI can be used in estimation of age among Indians. To compare SEE between both techniques, Modified Kvaals method shows lesser error when compared with CPCI.

Copy Right, IJAR, 2017,. All rights reserved.

Corresponding Author:- Pushpa.

Address:- Post graduate, MDS in Oral Medicine and Radiology, KLE V.K. Institute of Dental Sciences, Belagavi, Karnataka, India. 


\section{Introduction:-}

Age, or the time that a person has lived since birth, is an important cultural, religious and social phenomenon. Various stages of life are considered significant milestones such as a child's first birthday, the time to start school and the right to vote. To derive the chronological age of an individual a precise date of birth is required. This is conventionally confirmed by the birth registration documents which represent the starting point for the recognition and protection of every child's fundamental right to identity and existence. However, one in three developing countries has birth registration rates of less than $50 \%$. This has led to a variety of ways of assessing growth and development, one of which is the concept of maturity given by Tanner1975. When date of birth is uncertain, maturity markers may be used to estimate age. These maturity markers have been of considerable interest to physicians, anthropologists and dentists in order to correctly identify abnormalities.

Age estimation is one of several indicators employed to establish identity in forensic cases. Such estimations of living individuals are made for refugees or other persons who arrive in a country without acceptable identification papers and may require a verification of age, in order to be entitled to civil rights and/or social benefits in a modern society. ${ }^{1}$

Literature reports different morphological and radiological techniques for age assessment. Dental age estimation has gained acceptance because it is less variable when compared to other skeletal and sexual maturity indicators. ${ }^{2}$

Age estimation techniques are also useful for demonstrating substantive proof of birth records and age for immigrants (legal and otherwise) and those seeking retirement benefits. Therefore age estimation may be necessary for the living and diseased, and may apply to children, adolescents or adult age-groups. ${ }^{3}$

Dental changes for age estimation were undertaken by Schour and Massler and Gustafson. They evaluated dental age changes in children and adolescents by radiographs.

Later, morpho-histological technique and biochemical methods were put forward to assess dental ageing in adults. However, both techniques require tooth extraction, sectioning and further processing of dental tissues. Such invasive technique suit for post-mortem reconstructive procedure but in case of estimating age on living individuals, extraction in unethical. Hence, methods that do not require tooth extraction are also useful to evaluate age. These include clinical assessment of attrition, use of tooth colour as well as radiological methods. Such non-invasive methods can be used on the living and dead and do not require complex devices. ${ }^{3}$

Kvaal et al. ${ }^{1}$ were perhaps the first to develop a method for estimating age in adults using radiographs taken in situ. The method examined radiographs of 100 Norwegian adults.

Since the error of age estimation was higher with Kvaal's formula, a new age estimation formula was derived from obtained length and width ratios. The developed formula was then tested on an additional 100 subjects by Mohankumar et al and showed Standard error of estimated age by modified Kvaal's formula was less, when compared to standard error of estimated age of Kvaal and coauthors formula in sample Indian population. ${ }^{4}$

Study was carried out by RajandarTalabani et al to present a method for assessing chronological age based on the relationship between age and measurement of the tooth-coronal index (TCI) on mandibular first molar in an attempt to improve the precision and reliability of age estimations. ${ }^{5}$ Result showed the real age and the predicted age using TCI values were similar and the coefficients of correlation between actual age and predicted age were found to be very significantly high for first molars.

So this study was conducted to compare the accuracy between the techniques ie Kvaals with modified formula and coronal pulp cavity index.

Aim:-

To estimate the accuracy of age by using modified Kvaals formula and Coronal pulp cavity index. 


\section{Objectives:-}

1. To assess the accuracy of modified Kvaal's formula and Coronal pulp cavity index in estimating age among Indian population.

2. To compare the standard error of estimated age among both technique.

3. To establish the standard technique in assessing age among 5 groups.

After obtaining an informed consent, male individuals reporting to the Department of Oral Medicine and Radiology, KLE V.K. Institute of Dental Sciences, Belagavi, were included in the study.

Digital RVG of 100 individuals were taken.

Group A- Individuals of age 20-30years

Group B- Individuals of age 31-40years

Group C- Individuals of age 41-50years

Group D- Individuals of age 51-60years

Group E- Individuals of age 61-70years

\section{Inclusion criteria:-}

1. Individuals within the age group of 20-70 years.

2. Individuals having maxillary second premolar.

\section{Exclusion criteria:-}

1. Individuals with missing/ impacted maxillary second premolar

2. Carious tooth

3. Filled/prosthetically restored tooth

4. Malposed tooth

5. Tooth with periapical or pulpal pathologies

6. Tooth with morphological abnormalities, including attrition/abrasion/ erosion

7. Individuals having systemic diseases like Diabetes mellitus,

8. Hyper/Hypothyroidism,

9. Hyper/Hypoparathyroidism.

\section{Method of collection of data:-}

Informed consent was obtained from all individuals after explaining the procedure in language understandable to them.

Digital intraoral periapical radiographs were taken by using KODAK 8000 RVG machine with the exposure factors of $65 \mathrm{KVp}$ and $8 \mathrm{~mA}$ for $0.2 \mathrm{~s}$ for either right or left side of maxillary second premolar by paralleling technique.

Following measurements were made for Modified Kvaal's method using the KODAK Dental Imaging software: maximum tooth length, pulp length, root length on mesial side, pulp width at level 'a' (cementoenamel junction [CEJ]), level 'c' (midroot level), and level 'b' (midpoint of c and a), root width at level 'a' (CEJ), level 'c' (midroot level), and level 'b' (midpoint of $c$ and a).

In order to reduce the possible effects of variation in magnification and angulations of the radiographs, the following ratios were calculated:

1. Root length/tooth length $(T)$

2. Pulp length/tooth length $(R)$

3. Pulp length/root length $(P)$

4. Pulp width/root width at level a $(A)$

5. Pulp width/root width at level $\mathrm{b}(B)$

6. Pulp width/root width at level c $(C)$

7. Mean values of all ratios $(M)$

Mean value of width ratios from levels $\mathrm{b}$ and $\mathrm{c}(W)$

Mean value of length ratios $P$ and $R(L)$

Difference between $W$ and $L(W-L)$ 


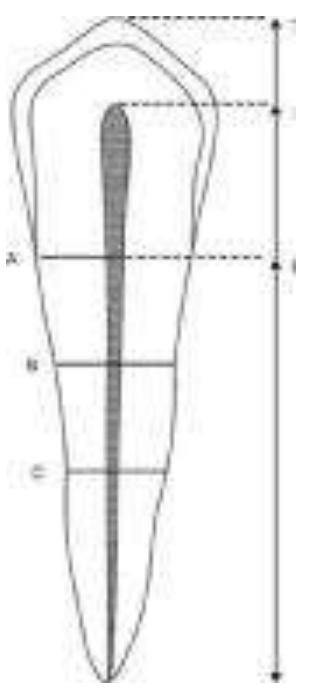

$\mathrm{T}$, maximum tooth length;

$\mathrm{R}$, root length on the mesial surface;

$\mathrm{P}$, maximum pulp length;

$A$, root and pulp width at cement-enamel junction (CEJ);

$\mathrm{B}$, root and pulp width at one-quarter of the root length from the CEJ;

$\mathrm{C}$, root and pulp width midway between CEJ and root apex

Age was assessed for all the individuals by using 2 predictors, where the mean of all ratios $(M)$ was taken as the first predictor, while the difference between the mean of the 2 width ratios and the mean of the 2 length ratios ( $W-L$ ) was taken as the second predictor.

\section{Coronal pulp cavity index:-}

On same tooth, measurements were performed using linear measurements. The CPCI, however, is an index based on two linear measurements and thus requires no further standardization of tooth size. The measurements undertaken are described below and illustrated in Figure.

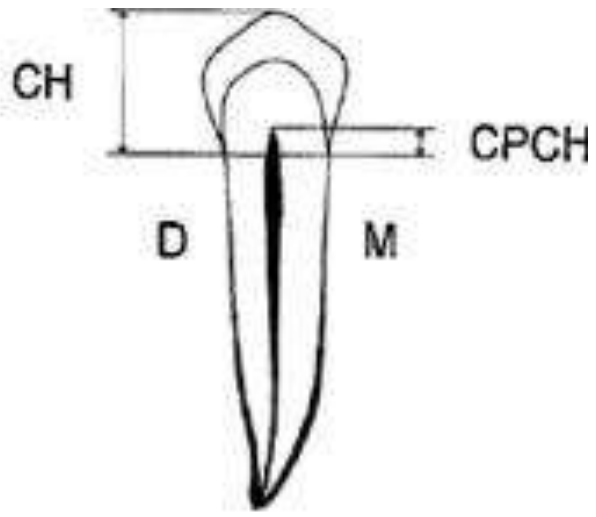

$\mathrm{CH}$ : height of the crown;

CPCH: coronal pulp cavity height. Adapted from Drusini et al.

A straight line was traced between the cemento-enamel junctions on the mesial and distal aspect. This defined the anatomical crown and the following measurements were acquired after Drusini et al.

1. Height of the crown $(\mathrm{CH})$ : measured vertically from the cervical margin to the tip of the highest cusp;

2. Height of the coronal pulp cavity $(\mathrm{CPCH})$ : measured vertically from the cervical line to the tip of the highest pulp horn;

3. Tooth coronal index $(\mathrm{TCI})=\mathrm{CPCH} X 100 / \mathrm{CH}$ 


\section{Statistical analysis:-}

1. Comparison of actual age with estimated age by CPCI and Kvaal methods by paired t test

2. Correlation Coefficients were used to estimate the actual age with estimated age by CPCI and Kvaal methods.

\section{Results:-}

The present study included 100 patients having maxillary second premolar in normal functional occlusion between age group of 20-70 years and divided into 5groups. Digital radiographs were taken in RVG and viewed using Kodak digital imaging software and converted to high resolution JPEG images. On the full size image, nine measurements were made on each individual's tooth based on Kvaal et al and Coronal pulp cavity index(Figure 1)

From the measurements, number of ratios were calculated in accordance with Kvaal et al. All ratios were calculated on an Excel worksheet(Microsoft Office 2007). To test for statistical significance, paired-t test was applied to compare actual age and estimated age on both CPCI and Kvaal's method as shown in (table 1) and shows significant p-value for estimated age by Kvaal's method.

(Table 2) shows comparison of error by estimated age by CPCI and Kvaal methods from actual age and reported significant p-value in Kvaal's method.

(Table 3) shows correlation coefficient between actual age with estimated age by CPCI and Kvaal's method and reported significant $\mathrm{p}$-value in both methods.

Comparison of actual age with estimated age by CPCI and Kvaal methods in different age groups is shown in(Graph 1). Among 5 groups, Modified Kvaal's formula can be applied among Group A, D, E and for Group B, C - CPCI can be applied.

Table 1:- Comparison of actual age with estimated age by TCI and Kvaal methods by paired t test

\begin{tabular}{|l|c|c|c|c|c|c|}
\hline Age & Mean & Std.Dv. & Mean Diff. & SD Diff. & Paired t & p-value \\
\hline Actual age & 44.88 & 16.12 & & & & \\
\hline Estimated age by TCI & 44.46 & 9.71 & 0.42 & 16.25 & 0.1841 & 0.8547 \\
\hline Actual age & 44.88 & 16.12 & & & & \\
\hline Estimated age by Kvaal & 52.27 & 14.57 & -7.39 & 3.74 & -13.9768 & $0.00001^{*}$ \\
\hline Estimated age by TCI & 44.46 & 9.71 & & & & \\
\hline Estimated age by Kvaal & 52.27 & 14.57 & -7.82 & 14.99 & -3.6867 & $0.0006^{*}$ \\
\hline
\end{tabular}

$* \mathrm{p}<0.05$

Table 2 :- Comparison of error by estimated age by TCI and Kvaal methods from actual age

\begin{tabular}{|l|c|c|c|c|c|c|}
\hline Variables & Mean & Std.Dv. & Mean & SD Diff. & Paired t & $\mathrm{p}$-value \\
\hline Error in TCI & & & Diff. & & & \\
\hline Error in Kvaal & 0.42 & 16.25 & & & & \\
\hline${ }^{*} \mathrm{p}<0.05$ & -7.39 & 3.74 & 7.82 & 14.99 & 3.6867 & $0.0006^{*}$ \\
\hline
\end{tabular}

Table 3:- Correlation coefficient between actual age with estimated age by TCI and Kvaal methods

\begin{tabular}{|l|c|c|c|}
\hline Variables & r-value & t-value & p-value \\
\hline Actual age with estimated age by TCI & 0.2876 & 2.0807 & $0.0428^{*}$ \\
\hline Actual age with estimated age by Kvaal methods & 0.9753 & 30.5863 & $0.00001^{*}$ \\
\hline Estimated age by TCI and Kvaal methods & 0.2896 & 2.0961 & $0.0414^{*}$ \\
\hline$* \mathrm{p}<0.05$ & & & \\
\hline
\end{tabular}

Graph:- Comparison of actual age with estimated age by TCI and Kvaal methods in different age groups. 


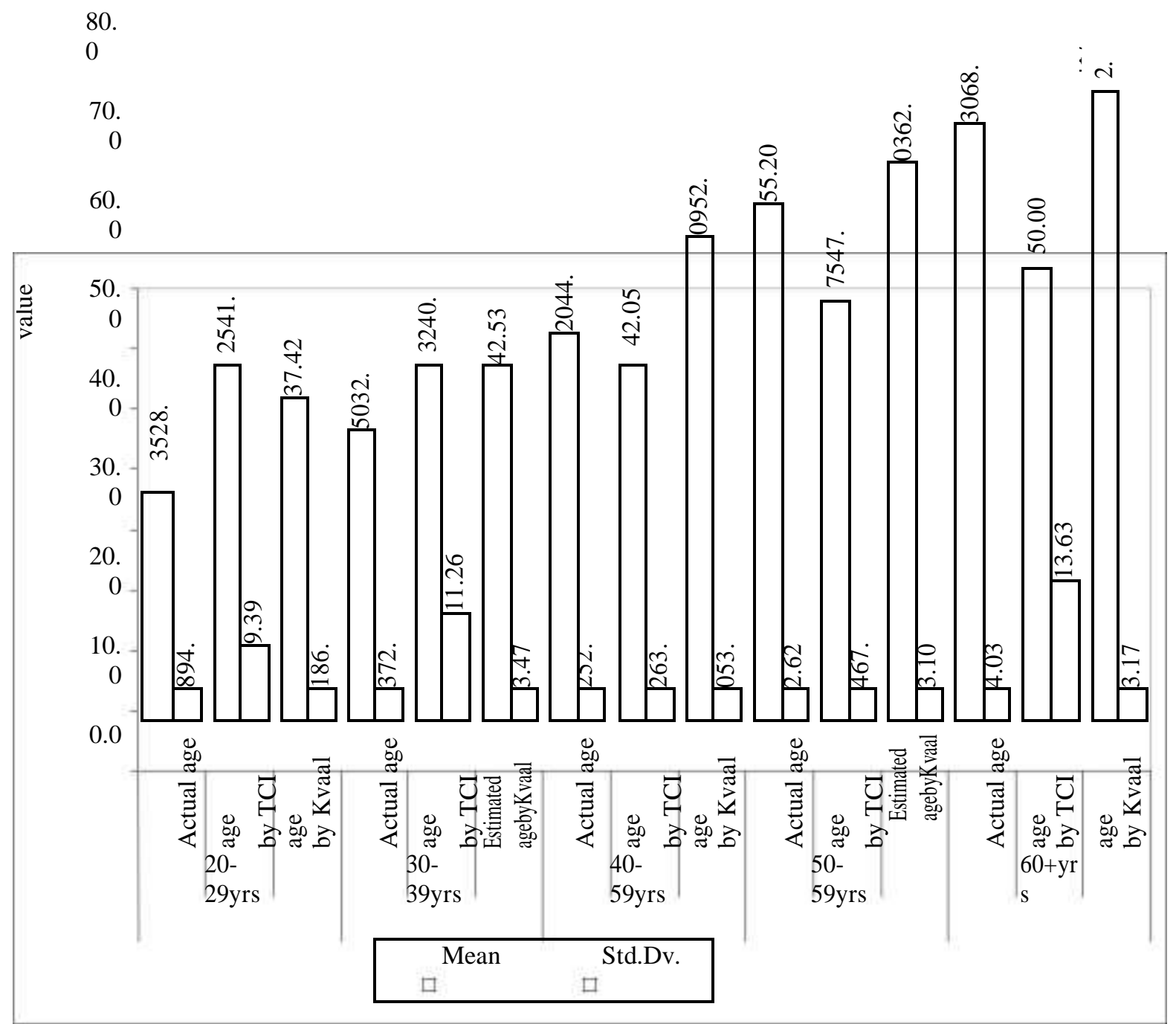




\section{Discussion:-}

Tooth is considered as a reliable body part for forensic age estimation since environment has minimal influence on it. Age estimation by radiographic evaluation is an established non-invasive technique for adults. ${ }^{9}$ Numerous accurate age estimation equations have been derived for western population, which when applied for Indian samples produced errors unacceptable in forensic age estimation. In order to enhance the accuracy of age prediction it is advocated to derive age estimating formulas specific to Indian population.

A study was conducted by Shruthi K Patil et al on estimation of age by Kvaal's technique in sample Indian population to establish the need for local Indian-based formulae.

The estimated age and chronological age were compared, less accurate results were found in sample Indian population. Modified Kvaal's formula was then developed. ${ }^{4}$ Hence in the present study, modified Kvaal's formula was used and showed less error on estimated age when compared to actual age.

The various studies that has been done to construct a formula for age estimation in Indian population has utilized radiographs of anterior teeth. ${ }^{9}$ But in elderly anterior teeth are often lost making age estimation difficult. ${ }^{9}$ Maxillary second premolar was chosen as Chandramala et al found "Upper second premolar" tooth as a good predictor of age, when taking "M" and "W-L" as the first and second predictors respectively. ${ }^{2}$ So in our study, maxillary second right/left premolar was used and showed significant results.

Digital radiography has various advantages over conventional techniques, such as instant image viewing, reduced radiation exposure, reusable plate etc. In the present study digital imaging was used as it produces a dynamic image in which visual characteristics of density and contrast can be manipulated after acquisition to meet specific diagnostic tasks and errors in exposure technique can be manipulated, which is not possible while using conventional film based intraoral radiographs. Moreover researchers like Bosmans et al ${ }^{10}$ have suggested the use of digital radiographs as an alternative to conventional radiography. Paralleling technique was used in this study for obtaining radiographs following Kvaal et all technique. This is important because if the radiographic film is at an angle to the mesiodistal plane of the tooth, the ratio between the pulp and root will be affected and the formulae derived will not be applicable. Paralleling technique also overcomes the disadvantages associated with bisecting technique namely, magnification, distortion, overlapping of anatomical structures, lack of reproducibility, partial images due to cone cut, overlapping of adjacent tooth crowns and foreshortening. 11

Ranjdar M. T et al conducted a study, a method for assessing chronological age based on the relationship between age and measurement of the tooth-coronal index (TCI) on mandibular first molar in an attempt to improve the precision and reliability of age estimations. ${ }^{5}$ Result showed the real age and the predicted age using TCI values were similar and the coefficients of correlation between actual age and predicted age were found to be very significantly high for first molars. Thus in the present study, the accuracy of age estimation was done by comparing modified Kvaal's formula with Coronal pulp cavity index and showed Modified Kvaal's formula can be applied among Group A, D, E and for Group B, C - TCI can be applied.

\section{Conclusion:-}

1. Modified Kvaal's formula and Coronal pulp cavity index can be used in estimation of age among Indians.

2. Modified Kvaal's formula is showing lesser error in estimated age when compared with Coronal pulp cavity index.

3. Among 5 groups, Group A, D, E - Modified Kvaal's formula can be applied, and for Group B, C - TCI can be applied.

Further studies are recommended with larger sample size, emphasizing on derivation and application of population specific as well as age group specific formulae to reduce the error of estimate, hence enhancing its reliability for use in forensic odontology. 


\section{References:-}

1. Sigrid I. Kvaal*“, Kristin M. Kolltveit”, Ib 0. Thomsenb,ToreSolheima Age estimation of adults from dental radiographs Forensic Science International 74 (1995) 175-185

2. RupaChandramala, Ridhima Sharma, Mubeen Khanand Anurag SrivastavaApplication of Kvaal's Technique of Age Estimation on Digital Panoramic Radiographs Dentistry 2:142. doi:10.4172/2161-1122.1000142

3. PreetiKanchan-Talreja, Ashith B. Acharya, Venkatesh G. NaikmasurAn assessment of the versatility of Kvaal's method of adultdental age estimation in Indians Archieves of Oral Biology 57 ( 2012 ) $277-284$

4. Shruthi K. Patil, Mohankumar K. P. Mandana Donoghue.: Estimation of age by Kvaal's technique in sample Indian population to establish the need for local Indian-based formulae Journal of Forensic Dental Sciences / September-December 2014 / Vol 6 / Issue 3

5. Age estimation using lower permanent first molars on a panoramic radiograph: A digital image analysis Ranjdar M. Talabani, Mohammed T. Baban and Mohammed A. Mahmood J Forensic Dent Sci. 2015 May-Aug; 7(2): $158-162$.

6. Ridhima Sharma and Anurag Srivastava Radiographic evaluation of dental age of adults using Kvaal's method J Forensic Dent Sci. 2010 Jan-Jun; 2(1): 22-26.

7. Piyush G. Limdiwala and J. S. Shah Age estimation by using dental radiographs J Forensic Dent Sci. 2013 JulDec; 5(2): 118-122.

8. ShalmiraKarkhanis, Peter Mack, Daniel Franklin Age estimation standards for a Western Australian population using the coronal pulp cavity index Forensic Science International 231 (2013) 412.e1-412.e6

9. Deepu George Mathew, S RajeshAdult forensic age estimation using mandibular first molar radiographs: A novel technique J Forensic Dent Sci. 2013 Jan-Jun; 5(1): 56-59. doi: 10.4103/0975-1475.114552

10. Nathalie Bosmans et al The application of Kvaal's dental age calculation technique on panoramic dental radiographs Forensic Science International 153 (2005) 208-212

11. Whaites E. Essentials of dental radiography and radiology. $3^{\text {rd }}$ edition, Churchill Livingstone; 2002. 